\title{
Stress Detection for PTSD via the StartleMart Game
}

\author{
Christoffer Holmgård*, Georgios N. Yannakakis ${ }^{\dagger}$, Karen-Inge Karstoft ${ }^{\ddagger}$, Henrik Steen Andersen ${ }^{\S}$ \\ ${ }^{*}$ Center for Computer Games Research, IT University of Copenhagen, Copenhagen, Denmark \\ ${ }^{\dagger}$ Institute for Digital Games, University of Malta, Msida, Malta \\ ${ }_{\ddagger}^{\ddagger}$ National Center for Psychotraumatology, University of Southern Denmark, Odense, Denmark \\ ${ }^{\S}$ Centre for Crisis and Disaster Psychiatry, Mental Health Services of the Capital Region of Denmark, Copenhagen, Denmark \\ *holmgard@itu.dk, ${ }^{\dagger}$ georgios.yannakakis@um.edu.mt, ${ }^{\ddagger}$ kikarstoft@ health.sdu.dk, ${ }^{\S}$ henrik.steen.andersen@ regionh.dk
}

\begin{abstract}
Computer games have recently shown promise as a diagnostic and treatment tool for psychiatric rehabilitation. This paper examines the positive impact of affect detection and advanced game technology on the treatment of mental diagnoses such as Post Traumatic Stress Disorder (PTSD). For that purpose, we couple game design and game technology with stress detection for the automatic profiling and the personalized treatment of PTSD via game-based exposure therapy and stress inoculation training. The PTSD treatment game we designed forces the player to go through various stressful experiences while a stress detection mechanism profiles the severity and type of PTSD via skin conductance responses to those in-game stress elicitors. The initial study and analysis of 14 PTSD-diagnosed veteran soldiers presented in this paper reveals clear correspondence between diagnostic standard measures of PTSD severity and skin conductance responses. Significant correlations between physiological responses and subjective evaluations of the stressfulness of experiences, represented as pairwise preferences, are also found. We conclude that this supports the use of the simulation as a relevant treatment tool for stress inoculation training. This points to future avenues of research toward discerning between degrees and types of PTSD using game-based diagnostic and treatment tools.
\end{abstract}

\section{INTRODUCTION}

Post Traumatic Stress Disorder (PTSD) is a psychiatric diagnosis describing an often severely disabling syndrome that is sometimes developed after being exposed to highly stressful situations. Veterans from military operations are a high-risk group for developing this syndrome [1]. A number of psychiatric treatments for PTSD are based on cognitivebehavioral approaches and include exposure therapy and stress inoculation training. Among the possible ways of treating PTSD computer games and virtual environments appear to have a great potential for eliciting stress in a controlled fashion and provide an immersive medium for PTSD treatment facilitating exposure therapy and stress inoculation training.

In this paper we investigate the usefulness of game design incorporating affect detection to support the psychiatric treatment of PTSD-diagnosed veteran soldiers. For that purpose, we designed and developed a game - StartleMart — that expands upon existing principles of PTSD treatment techniques with game mechanics and uses stress detection to infer the user's physiological responses to in-game events. In this initial study we run a test with 14 veterans diagnosed with PTSD and examine the impact of their PTSD psychiatric profile on the arousal responses - measured via skin conductance (SC) - they manifest through in-game stress elicitors. In addition we examine the relationship between self-reported stress levels and SC signal features. Results show that physiological responses correlate with both PTSD profile features and selfreports of stress, supporting the relevance of the StartleMart game for PTSD diagnosis and treatment.

This work is novel as it uniquely combines real-time stress detection with a game (virtual) environment aimed at PTSD treatment. Diverging from and innovating upon earlier work in the use of simulations for treating PTSD [2], we argue that simulating everyday-life situations can help PTSD patients improve their functioning in everyday tasks with direct benefits to their quality of life as a form of stress inoculation training [3]. The present study expands on previous research and approaches by constructing a desensitization and exposure paradigm consisting of a virtual world taking place in a homelike setting with integrated game mechanics. The result is a hitherto unexplored midpoint between mediated and in vivo exposure paradigms aimed at addressing issues in the everydaylife of the patient.We believe that by interweaving appropriate game design and efficient stress profiling we can provide a personalized therapeutic environment that allows therapists, for the first time, to detect and address common PTSD symptoms across individuals with varying etiologies behind their PTSD. For instance, a veteran soldier and an assault victim may exhibit similar responses to stressful everyday-life situations and a simulation addressing these situations would be relevant to both.

\section{BACKGROUND: StRESS DETECTION AND PTSD TREATMENT}

This section covers related work on affect detection, the relationship between stress disorders and physiology, treatment types for PTSD and our approach to PTSD diagnosis and treatment, and, finally, the use of virtual environments and computer games for the treatment of PTSD.

\section{A. Stress Detection}

A wide range of approaches exist for capturing stress using physiological, behavioral, and self-report data or combinations thereof. Earlier work on stress detection [4] has demonstrated how features extracted from raw physiological signals can be used to discern between a variety of emotional states in general [5] and in games [6]. SC has been identified as a useful indicator of stress elicited from tasks [7], [8] and with soldiers [9]. Innervation of the sweat glands is caused solely by the autonomic nervous system, making it a well suited source for measuring specifically arousal and, by extension, stress [10]. Thus, SC is an obvious physiological indicator of player stress. It has been indicated that it is necessary to mediate 
the interpretation of physiological measurement data with information from self reports of phenomenal experience [11]. For this reason we also use self-reports to collect information about the stressfulness of using StartleMart. Because selfreports have been shown to be unstable over time and hard to anchor to fixed scales between sessions [12], we treat self-reports as expressions of preference rather than directly comparable indications of subjective experience. By collecting physiological responses synchronously with log data from the game environment, we build on previous attempts to use affective computing to link responses to presented stimuli with PTSD symptom severity [2], [13].

\section{B. Physiology of PTSD}

In mediated stimulus exposure paradigms, PTSD-patients exhibit physiological responses to stressful visual and auditive stimuli that are significantly different from the responses of non-patients [9]. Their responses are generally characterized by high sympathetic activity as measured by SC. In experimental studies, slower SC habituation, elevated resting SC, and greater SC responses to startling stimuli, have been found to be robust identifying characteristics of PTSD-patients [14]. This indicates the higher base levels of arousal and heightened sensitivity to stress that are typical of the disorder. It has been suggested that these differences could be used to support diagnostic differentiation between PTSD patients and nonpatients as well as between different degrees of PTSD symptom severity [15] guiding treatment strategies or allowing for adaptive treatment tools [2]. In the present study we innovate by investigating the relationship between PTSD profiles, selfreports of stress and SC signal features of arousal.

\section{Treating Stress Disorders}

Two well-known treatment approaches for PTSD are the cognitive-behavioral therapy techniques of exposure therapy and stress inoculation training. In exposure therapy, the therapist confronts the patient with anxiety provoking stimuli in a controlled setting in order to extinguish reactions to the stimuli and/or allow the patient to reprocess the memories cued by the stimuli. Three common variations are the use of real life stimuli i.e. in vivo, representing stimuli via media i.e. mediated, or having the patient imagine the stress provoking situations and thus self-generate the stimuli i.e. imaginal [3]. In stress inoculation training, the therapist exposes the patient to stimuli and situations that are not directly linked to the original trauma of the patient, but that cause problematic anxiety responses that are difficult for the patient to cope with [3]. In the present study we utilize StartleMart as a game facilitator of exposure therapy and stress inoculation training.

\section{Games for Mental Health}

Games and game-like worlds have successfully been used as mental health interventions by appropriating commercial games [16] and by developing specialized solutions [17]. Earlier research has demonstrated the usefulness of virtual environments for treating veterans' PTSD with virtual reality therapy, an extension of exposure therapy [2], [18]. Virtual reality therapy most often focuses on exposing the patient to the original stressful, traumatizing situation, in the vain of classic exposure therapy, rather than appropriating principles from stress inoculation training. Notable examples are the Virtual Iraq and Virtual Afghanistan applications that show promising results in clinical testing [19], [20]. In the StartleMart game, instead, we adopt a hybrid approach coupling exposure therapy and stress inoculation training which is informed by real-time stress detection for personalized treatment.

\section{The StartleMart Game FOr PTSD Treatment}

The design of StartleMart is guided by the joint efforts of a multidisciplinary team consisting of researchers and practitioners from the fields of psychotraumatology, game design and game technology. Informed by the mental health professionals on the design team, we assume that being able to expose patients to simulations of mundane, but stressful, situations will be effective for diagnosis and treatment while maintaining flexibility in terms of possible patient groups that can benefit from the game. The task of going shopping in a supermarket is as a common situation that is severely challenging to many patients suffering from PTSD [21]. Supermarkets are highly stimulating environments with social interactions and unpredictable auditive and visual experiences which PTSD patients find stressful; some to the extent that they avoid going shopping or only do so with a helper present for emotional support. Consequently, the game is built to primarily take place in a virtual supermarket (see Fig. 1). Since many veterans suffering from PTSD report re-experiencing memories of the originally traumatizing situation when cued by elements in the environment, the game includes short flashbacks (see Fig. 1). These momentarily change the environment of the game to an Afghan theater of operations, before changing back into the supermarket, embedding elements of exposure therapy [3] in the stress inoculation training [3] approach ${ }^{1}$. We assume that the player's level of immersion into the game environment will influence the extent to which he responds to the stressors in the game [22]. To support immersion, the simulation is presented as a first-person-shopper. The player starts at the entrance of the supermarket, navigates through the supermarket collecting items on the list and concludes a mission by standing in line and paying at the cash register. The player can move freely around the supermarket and collect or activate goods or items. In order to ensure that the player experiences as much of the supermarket as possible, the objective of the game is to collect a number of goods presented on an on-screen shopping list within a given time frame. The items to collect and the remaining time are displayed on screen during the game (see top-right of the left-hand images in Fig. 1). The goods are placed in locations that make it probable that the player will be exposed to all sections of the supermarket if he manages to collect all items.

\section{A. Game Stressors}

The supermarket environment includes a number of stressors that aim at eliciting stress in the player. These are designed around three typical symptoms of PTSD, namely fear-avoidance behavior, hyper-arousal (i.e. heightened startle response), and re-experiencing of traumatic events triggered by an outside stimulus or general stress [3]. Stressors targeting fear-avoidance behavior include the following design

\footnotetext{
${ }^{1}$ As the flashbacks constitute a minor part of the content they can easily be omitted or exchanged for groups of patients that are not war veterans.
} 


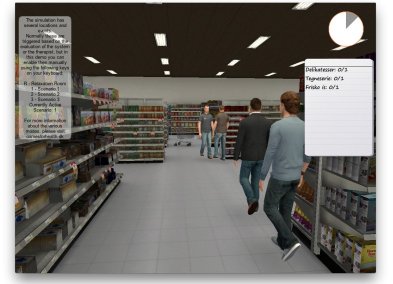

(a) Sound of ventilator blowing overhead.

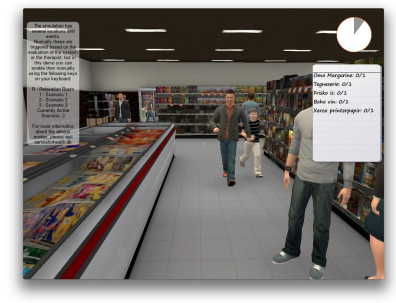

(c) Man walking angrily toward player.

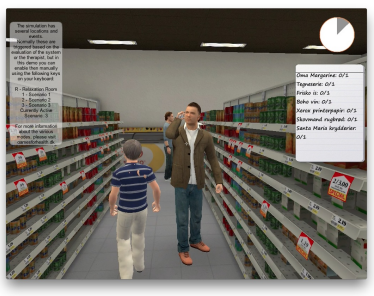

(e) Man staring at player.

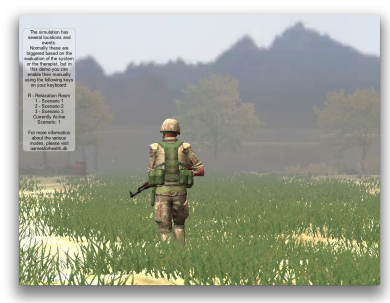

(b) Sound of wind blowing.

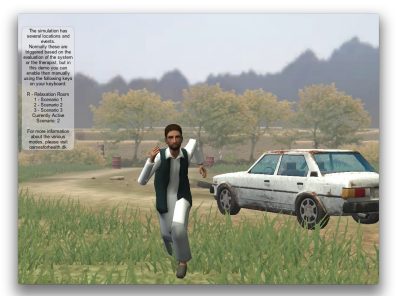

(d) Afghan running toward player.

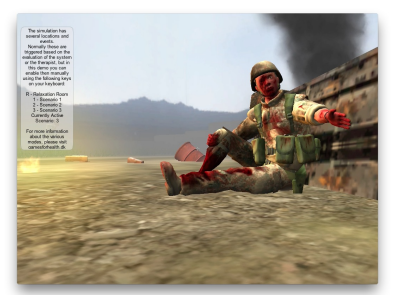

(f) Wounded soldier staring at player.
Fig. 1: The three flashbacks of the game $(b, d, f)$ and the immediately preceding supermarket scenes (a, c, e). Elements of the supermarket bleed into the flashbacks, simulating reexperience.

elements: The layout of the supermarket is designed to include hidden angles and preventing the player from attaining a full overview of the location. An aisle is blocked by a shopping cart making it difficult for the player to pass. Non-PlayerCharacters (NPCs), adults as well as children, wander around the supermarket, sometimes blocking the way of the player. Two NPCs, engaged in conversation, will stop talking and stare at the player if he approaches. An NPC walks angrily down the aisle toward the player, expressing aggression through his body-language. A family of NPCs are engaged in a discussion, the father is scolding the child aggressively. An NPC pockets goods from the shelves of the supermarket. Stressors targeting hyper-arousal include a dog barking at the entrance to the supermarket and the sound of crashes and glass breaking suddenly playing at random locations in the supermarket. Stressors targeting re-experiencing are included in the form of three different flashbacks. Their purpose is to elicit the feeling of recalling and re-experiencing a traumatic memory. Only one is shown per mission. In the first flashback the player is walking on a foot patrol in a typical Afghan theater of operations (see Fig. 1b). In the second flashback the player sees a man, apparently of Afghan origin, running directly toward him (see Fig. 1d). In the third flashback the player sees a fellow soldier hit by an explosion, clutching the remains of his leg (see Fig. 1f). The game features three different configurations

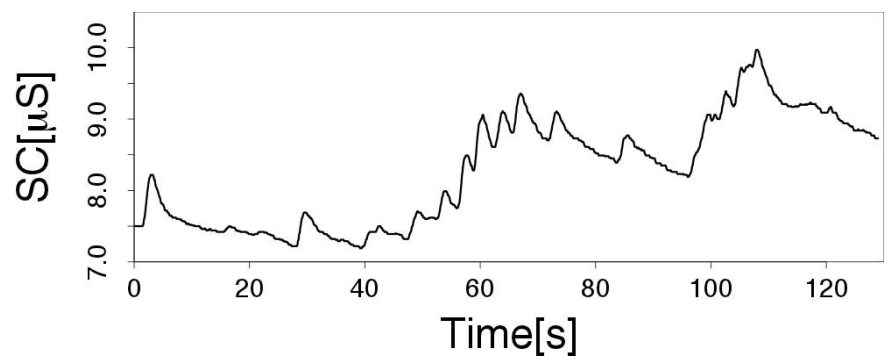

Fig. 2: An example of an SC signal recorded from a single game session: Subject no. 5, game session 3 (high stress intensity).

of missions assumed to elicit stress to different degrees. The missions vary in terms of the number of items the player must collect and the apparent threat in the presented flashback. We assume that increasing the number of items the player has to collect and increasing the degree of threatening content in the flashbacks will increase the stressfulness of the experience. The missions are played consecutively going from the least stressful to the most stressful.

\section{Experimental Protocol and Data Collection}

In this section we provide details about the participants of our experiment and the experimental protocol followed for the clinical trials of the game. Fourteen male PTSD patients, veterans from Danish military operations in Afghanistan, are included in the study presented in this paper. The participants are in psychiatric treatment for PTSD and qualify for the PTSD diagnosis. All subjects in the sample are medicated with Selective Serotonin Re-uptake Inhibitors (SSRI) which is known to generally lower sympathetic activity and in particular SC [23]. This clearly ads a challenge to the detection of SC stress responses to game stimuli since patients are expected to manifest responses that are pharmacologically suppressed to an unknown degree. Our approach, thus, is based on a within-subject analysis to eliminate such effects. Each patient participates in the experiment twice, engaging in a total of 6 game play sessions, 3 per participation (11 patients have participated in both sessions, while 3 participated in the first session only). The experimenters, trained psychologists, welcome the participant, complete a diagnostic interview with the patient and collect various instances of demographic and background data from either the patient himself or the patient's medical records (see section $\mathrm{V}-\mathrm{A}$ ). The participant is introduced to the experimental setup and seated in front of the controls and monitor. The biofeedback device is attached to the participant's fingertips (see more details in section IV-A), and a brief introduction to the game rules and how to control game is given. Following a short waiting period, collecting baseline SC data, the participant is asked to play three sessions of the game. Subjective data (self-reports) are collected over the course of the experiment (see section V-B). Finally, the experimenter debriefs the participant, responding to any concerns or issues the patient might have.

\section{A. Physiological Sensors and Setup}

For continuous measurement of SC the IOM biofeedback device $^{2}$ is used. The IOM biofeedback device samples SC

\footnotetext{
${ }^{2}$ http://www.wilddivine.com/
} 
TABLE I: PTSD profile features

\begin{tabular}{c|c|c|c}
\hline \hline Feature & Average & Standard deviation & Range \\
\hline Age & 26.8 & 2.5 & $22-32$ \\
PCL & 58.0 & 4.9 & $50-65$ \\
$N_{\text {dep }}$ & 1.77 & 0.67 & $1-3$ \\
$N_{\text {day }}$ & 1001.2 & 432.4 & $113-1685$ \\
\hline \hline
\end{tabular}

at a rate of $300 \mathrm{~Hz}$ and down-samples them to $30 \mathrm{~Hz}$ in firmware before transmitting them to the recording computer. The device's measuring electrodes are attached dryly to the distal phalanges of the little and middle fingers of the patient's non-dominant hand. A sensor measuring blood volume pulse is attached to the ring finger, but is not used for the study and analysis presented here. To ensure maximum exposure to the content the game is presented on a 25 " LCD monitor placed roughly $35 \mathrm{~cm}$ from the face of the patient. To provide auditive stimulation, while still allowing the player to communicate with the experimenter, supra-aural headphones are used to deliver the sounds of the game. The audio level is adjusted to be experienced subjectively as loud, but pleasant. Since frustration with the control scheme of the game might introduce unwanted variation and artifacts to the results of the experiment [24] the game is configured to use standard controls for first-person-perspective computer games which should be familiar to most patients. The mouse, operated with the patient's dominant hand, controls the perspective and the keyboard controls movement. To minimize the risk of movement artifacts in the physiological readings, patients operate the keyboard (W, A, S, D or arrow keys) with only the index finger of their non-dominant hand, keeping the other fingers still. An example of a SC signal collected from a single session is illustrated in Fig. 2.

\section{USER Data Features}

This section details the three types of data obtained from, or extracted for, each experiment participant considered in this study. These include the PTSD profile of the patient, the subjective self-reports of stress during the experiment and the the set of features extracted from the SC signal.

\section{A. PTSD profile}

Each participant is subjected to the PTSD Module of the Structured Clinical Interview for the DSM (SCID) [25] and completes the military version of the PTSD ChecklistIV (PCL-M) [26], a 17-item questionnaire that yields a PTSD symptom severity score in the interval $17-85$. Then all patients are profiled in terms of age, PTSD checklist score $P C L$, number of deployments (i.e. war missions) experienced $N_{d e p}$, and the number of days since their return from their latest deployment $N_{d a y}$. The average, standard deviation and range values of the PTSD profile features across all 14 patients are presented in Table I. For the veteran PTSD patients, traumatized by experiences during deployment in this study, we assume that $N_{d a y}$ may be considered an adequately precise measure of the time passed since the traumatizing experience. The deployment situation as a whole may be considered a highly stressful experience and as such part of the traumatizing situation. This means that the age of the trauma for all purposes here is assumed to be equivalent to $N_{d a y}$.

\section{B. Self-Reports of Stress}

Before, immediately after, and following a short break after each of the three sessions, the patient is asked to provide a rating of his subjectively experienced stress level on the Subjective Units of Distress Scale (SUDS) [27] in a range from 0 to 100 with 0 representing complete absence of stress and 100 representing the most stressful experience the patient can recall.

\section{Features extracted from Skin Conductance}

SC features are extracted from complete game sessions. Session data is procedurally and visually inspected for outliers and other indications of artifacts. Session data instances containing artifacts are either reconstructed, if possible, or removed from the data set. Following this data cleaning process - that removed 7 (9\%) of all possible 75 game sessions resulting in a total of $68(91 \%)$ sessions - all signals are adjusted for baseline readings, subtracting the individual session mean baseline value from the raw signal. Prior to feature extraction all signals are normalized via min-max normalization within individuals and across sessions from the same day. In order to account for any day-variation effects, signals from the same patients, but taken on different days, are treated as separate individuals. In accordance with recommendations from earlier studies on SC signal processing [5], [6], [28], a number of features of the SC signal are extracted: Mean SC value $S C_{\bar{x}}$, standard deviation of the $\mathrm{SC}$ signal $S C_{\sigma}$, minimum $\mathrm{SC}$ value $S C_{\min }$, maximum SC value $S C_{\max }$, the difference between the maximum and minimum $\mathrm{SC}$ value $S C_{\max -\min }$, the correlation between recording time $t$ and SC values $R_{S C t}$, the value of the first SC sample $S_{\text {init }}$, the value of the final SC sample $S_{\text {last }}$, the difference and absolute difference between final and first SC value $S C_{\text {last-init }}$ and $\left|S C_{\text {last-init }}\right|$, the time of the minimum SC value $t_{S C_{m i n}}$, the time of the maximum SC value $t_{S C_{m a x}}$, the time $t$ difference between the minimum and maximum SC values $\left|t_{S C_{\max \text { min }} \mid}\right|$ the means of the absolute values of the first and second differences of the SC signal $S C_{\left|\delta_{1}\right|}$ and $S C_{\left|\delta_{2}\right|}$. An uncommonly used feature: the mean of the absolute first difference of the absolute first difference $\left|S C_{\delta_{\delta}}\right|$ is added in an attempt to describe the tendency toward weak habituation in the signal.

\section{RESULTS}

We assume there is a relationship between PTSD profile and manifestations of stress on the SC signal and, thus, investigate the effects of PTSD profile features on SC signals. We also investigate the relationship between self-reported levels of stress and features of the SC signal. On that basis, we follow a correlation analysis for examining both relationships and present the key findings of this initial analysis.

\section{A. Correlation analysis between PTSD profile and SC features}

To investigate the impact of a PTSD profile to manifestations of stress via SC we compute correlations between the two sets of features using Spearman's rank correlation coefficient $\rho$ [29]. The results are presented in Table II. Results suggest that patients suffering from more severe degrees of PTSD (higher PCL values) respond with higher $S C_{\max }$ and a higher increase across the sessions as indicated by $S C_{\text {last-init }}$. 
TABLE II: Correlations $\rho$ between SC signal and PTSD profile features in the left section of the table. Correlations $c(z)$ between SC signal and self-reported stress in the right section of the table. Statistically significant correlations appear in bold (p-value $<0.05)$ and italics (p-value $<0.10)$.

\begin{tabular}{c|rrrr|rr}
\hline \hline & Age & PCL & $N_{\text {dep }}$ & $N_{\text {day }}$ & $\begin{array}{c}\text { Day } \\
\text { pairs }\end{array}$ & $\begin{array}{c}\text { Adjac. } \\
\text { pairs }\end{array}$ \\
\hline$S C_{\bar{x}}$ & 0.10 & 0.10 & 0.01 & 0.08 & 0.15 & 0.14 \\
$S C_{\text {max }}$ & 0.22 & $\mathbf{0 . 2 9}$ & 0.05 & -0.25 & -0.15 & 0.00 \\
$S C_{\text {min }}$ & -0.16 & 0.03 & $-\mathbf{0 . 3 1}$ & 0.05 & -0.15 & 0.00 \\
$S C_{\text {max-min }}$ & 0.23 & 0.24 & 0.13 & -0.26 & $-\mathbf{0 . 2 5}$ & $-\mathbf{0 . 1 9}$ \\
$S C_{\sigma}$ & 0.26 & 0.17 & 0.13 & -0.23 & -0.15 & 0.00 \\
$R_{S C t}$ & 0.10 & 0.02 & 0.15 & -0.06 & 0.15 & 0.14 \\
$S C_{\text {init }}$ & 0.11 & 0.08 & -0.13 & 0.10 & $\mathbf{0 . 2 5}$ & 0.10 \\
$S C_{\text {last }}$ & 0.08 & $\mathbf{0 . 3 5}$ & -0.17 & $-\mathbf{0 . 3 0}$ & -0.02 & -0.05 \\
$S C_{\text {last-init }}$ & -0.08 & $\mathbf{0 . 3 1}$ & -0.03 & -0.25 & -0.02 & 0 \\
$\left|S C_{\text {last-init }}\right|$ & 0.09 & $\mathbf{0 . 3 2}$ & -0.01 & $-\mathbf{0 . 3 5}$ & -0.02 & 0.05 \\
$t_{S C_{\text {min }}}$ & 0.06 & 0.02 & -0.13 & -0.02 & -0.12 & -0.10 \\
$t_{S C_{\text {max }}}$ & -0.17 & 0.06 & 0.10 & -0.12 & -0.02 & 0.00 \\
$\mid t_{S C_{\text {max }- \text { min }} \mid}$ & -0.04 & -0.07 & 0.11 & -0.15 & 0.08 & 0.05 \\
$S C_{\left|\delta_{1}\right|}$ & 0.15 & $\mathbf{0 . 2 9}$ & 0.13 & -0.26 & -0.12 & -0.14 \\
$S C_{\left|\delta_{2}\right|}$ & 0.15 & $\mathbf{0 . 2 8}$ & 0.14 & -0.25 & -0.12 & -0.14 \\
$S C_{\left|\delta_{\delta}\right|}$ & 0.15 & $\mathbf{0 . 2 8}$ & 0.14 & -0.25 & -0.02 & 0.00 \\
\hline \hline
\end{tabular}

This corresponds to findings that PTSD patients are more responsive to stressful stimuli. They also complete the session with a higher $S C_{\text {last }}$ which corresponds to findings that PTSD patients are more responsive and habituate slower than nonpatients. Patients with more severe PTSD exhibit higher values of all typical measures of local variation. The correlations between PCL and $S C_{\left|\delta_{1}\right|}, S C_{\left|\delta_{2}\right|}$ indicate that patients with more severe PTSD exhibit more variation. We hypothesize this is due to the relation between the severity of the syndrome and the hyper-responsiveness and hyper-arousal of the patient, meaning the patient responds more often to stimuli in the game. $S C_{\left|\delta_{\delta}\right|}$ also correlates with symptom severity suggesting PTSD patients' slower habituation compared to non-patients [14]. Significant positive correlation is observed between $N_{d e p}$ and $S C_{\min }$. No clear explanation can be given for this, since more deployments should mean a higher degree of exposure to potentially highly stressful situations, but it should be noted that the range of the number of deployments in the sample is limited to $1--3$. One could speculate that individuals who were only diagnosed with PTSD after several deployments were less susceptible to contracting the hyper-aroused state of PTSD. It would follow that they would exhibit lower SC bounds than their more susceptible colleagues, but the explanation remains speculation. A negative correlation is observed between $N_{d a y}$ and the last SC value recorded in session; PTSD symptoms typically abate as a function of time [3], so this relation matches the literature on PTSD. The literature also matches the relation between $N_{d a y}$ and $P C L$ : $P C L$ and $N_{d a y}$ correlate negatively $(\rho=-0.51, p<0.01)$ indicating the symptom severity decreases over time. It seems plausible that $N_{d a y}$ is an inverse indicator of symptom severity and that less severe cases of PTSD exhibit lower bounds of SC, most likely due to a less elevated mean SC level and faster (closer to normal) habituation. Altogether, we argue the results indicate a positive relationship between symptom severity and physiological stress responses to StartleMart.

\section{B. Correlation analysis between self-reports and SC features}

To investigate relations between self-reported levels of stress and signal features another measure of correlation is computed. As noted in section II-A there is reason to believe that pair-wise preference analysis is a useful approach for examining self-reports. For this purpose, preference pairs are created with each pair containing a self-report value and a feature value. Correlation values are calculated via the following test statistic [30]

$$
c(z)=\sum_{i=1}^{N_{s}}\left\{z_{i} / N_{s}\right\}
$$

where $z_{i}=1$ if the highest stress value is from the same observation of the pair as the highest feature value; otherwise $z_{i}=-1$. In cases where the SUDS ratings are equal the stress preference pair is considered ambiguous and discarded. The relations between the self-reported SUDS ratings collected from the patients are expected to become increasingly vague over time. This, in turn, affects the quality of self-reported ratings. Episodic memory traces that form the basis of self reports fade over time, but the precise rate at which this memory decay occurs is unknown in this case and most likely individual [31]. Ideally, memory decay is so slow that the patient will have a clear feeling of the first session when rating the final session, but it is possible that only comparisons between immediately adjacent sessions are valid. To account for this uncertainty, two different correlation analyses are attempted: one combining data from sessions on the same day and one combining data only from sessions that took place immediately adjacent to each other. The results are included in Table II. Two significant effects are identified across the two approaches: A negative correlation between self-reports of stress and the range of the SC signal $\left(S C_{\max -\min }\right)$ and a positive correlation between reported stress and initial SC values. Both effects are consistent with the fact that patients with severe PTSD symptoms exhibit high SC values and weaker habituation. This means their SC values stay higher and their signals are subject to quick stabilization at the individually higher baseline. The correlations indicate that patients feeling stressed by interacting with StartleMart exhibit matching physiological responses and supports the relevance of the game to the target group.

\section{DISCUSSION}

The $P C L$ score of the patients served as the first measure of ground truth describing symptom severity in this study. The $P C L$ instrument is well-validated and the de facto standard for PTSD severity screening [3], but is nonetheless based on self-reports of personal experience retrieved from memory. This is an inherent weakness of the presented study, but one we suspect is innate and difficult to overcome in any study involving a syndrome defined partially by personal experience. The negative correlation between PCL values and $N_{d a y}$, which matches expectations according to the literature, strengthens the validity of the measure. The second measure of ground truth is the SUDS values collected during the gameplay sessions. These are subject to the concerns described in sections II-A and VI-B, but these concerns are sought mediated by the use of pair-wise preferences as the basis for correlation. In Table II negative correlations are present between selfreports and $S C_{\max }$ and $S C_{\min }$ when pairs are constructed 
across all sessions in a day. These correlations trend in the opposite direction of what we would expect from theory. When pairs are limited to adjacent sessions these effects disappear and only effects matching expectations from theory remain. We consider this an indication of the psychometric properties of self-reports of stress. Future work using StartleMart might benefit from including stress evaluations as preferences at the report level. The analyses presented in this paper are limited to correlating features. Recent work in the literature [8], [32] describes how applications of non-linear techniques of analysis and machine learning can support stress detection and the data set described here could advantageously be analyzed by these methods in the future. Finally, the application of SC signal deconvolution could allow us to separate tonic and phasic components of the SC signal, identifying phasic drivers underlying responses to in-game events [33]. This could allow us to develop personalized, event-based PTSD profiles that integrate information from the simulation context into the stress detection process.

\section{CONCLUSION}

The results of the analyses in this paper indicate that physiological responses to StartleMart are highly correlated with PTSD symptom severity and subjective experience expressed through self-reports of stress. That StartleMart elicits stress responses with PTSD patients lends credence to the general idea of using game-based stimuli of every-day life situations for stress inoculation training for PTSD patients. However, any treatment efficacy is unknown at this point and would require a randomized study. Nonetheless, the fact that physiological responses seem to scale with measures of symptom severity, self-reports and an indicator of recovery over time, indicates a promise to using StartleMart for diagnosis and treatment of PTSD. Future work will focus on leveraging these findings to refine profiling and adaptive game-based solutions supporting diagnosis and treatment in psychiatric work.

\section{ACKNOWLEDGMENTS}

This research was supported by the Danish Council for Technology and Innovation under the Games for Health project and by the FP7 ICT project SIREN (project no: 258453).

\section{REFERENCES}

[1] C. W. Hoge et al., "Combat duty in iraq and afghanistan, mental health problems, and barriers to care," New England Journal of Medicine, vol. 351 , no. 1 , pp. 13-22, 2004

[2] D. P. Wood et al., "Reality graded exposure therapy with physiological monitoring for the treatment of combat related post traumatic stress disorder: a pilot study." Studies in health technology and informatics, vol. 163, p. 696, 2011.

[3] E. B. Foa et al., Effective treatments for PTSD, practice guidelines from the International Society for Traumatic Stress Studies, 2nd ed. New York: Guilford Press, 2009.

[4] R. A. Calvo and S. D'Mello, "Affect detection: An interdisciplinary review of models, methods, and their applications," IEEE Transactions on Affective Computing, vol. 1, no. 1, pp. 18-37, 2010.

[5] R. W. Picard, E. Vyzas, and J. Healey, "Toward machine emotional intelligence: Analysis of affective physiological state," IEEE PAMI, vol. 23, no. 10, pp. 1175-1191, 2001

[6] H. P. Martínez, M. Garbarino, and G. N. Yannakakis, "Generic physiological features as predictors of player experience," in ACII. Springer, 2011, pp. 267-276.
[7] J. A. Healey and R. W. Picard, "Detecting stress during real-world driving tasks using physiological sensors," IEEE ITS, vol. 6, no. 2, pp. 156-166, 2005.

[8] J. Hernandez, R. R. Morris, and R. W. Picard, "Call center stress recognition with person-specific models," in ACII. Springer, 2011, pp. $125-134$

[9] C. Perala, "Galvanic skin response as a measure of soldier stress," DTIC Document, Tech. Rep., 2007.

[10] W. Boucsein, Electrodermal activity. Springer Verlag, 2011.

[11] S. Tognetti et al., "The affective triad: stimuli, questionnaires, and measurements," in ACII. Springer, 2011, pp. 101-110.

[12] G. N. Yannakakis and J. Hallam, "Ranking vs. preference: a comparative study of self-reporting," in ACII. Springer, 2011, pp. 437-446.

[13] S. Popović et al. (2009) Stress inoculation training supported by physiology-driven adaptive virtual reality stimulation.

[14] N. Pole, "The psychophysiology of posttraumatic stress disorder: A meta-analysis." Psychological Bulletin, vol. 133, no. 5, p. 725, 2007.

[15] J. Blechert et al., "Autonomic and respiratory characteristics of posttraumatic stress disorder and panic disorder," Psychosomatic Medicine, vol. 69, no. 9, pp. 935-943, 2007.

[16] E. A. Holmes et al., "Can playing the computer game "tetris" reduce the build-up of flashbacks for trauma?" PLoS One, vol. 4, no. 1, 2009.

[17] M. E. Hoque et al., "Exploring speech therapy games with children on the autism spectrum," 2009.

[18] T. Parsons and A. Rizzo, "Affective outcomes of virtual reality exposure therapy for anxiety and specific phobias: a meta-analysis," Journal of Behav. Therapy and Exp. Psychiatry, vol. 39, no. 3, pp. 250-261, 2008.

[19] G. Reger et al., "Effectiveness of virtual reality exposure therapy for active duty soldiers in a military mental health clinic," Journal of traumatic stress, vol. 24, no. 1, pp. 93-96, 2011.

[20] A. Rizzo et al., "Virtual reality exposure therapy for combat-related ptsd," Post-Traumatic Stress Disorder, pp. 375-399, 2009.

[21] T. B. Kashdan et al., "Everyday strivings in war veterans with posttraumatic stress disorder: Suffering from a hyper-focus on avoidance and emotion regulation," Behav. therapy, vol. 41, no. 3, pp. 350-363, 2010.

[22] A. McMahan, "Immersion, engagement and presence," The video game theory reader, pp. 67-86, 2003.

[23] M. Siepmann et al., "Effects of sertraline on autonomic and cognitive functions in healthy volunteers," Psychopharmacology, vol. 168, no. 3, pp. 293-298, 2003.

[24] G. N. Yannakakis, H. P. Martínez, and A. Jhala, "Towards affective camera control in games," User Modeling and User-Adapted Interaction, vol. 20, no. 4, pp. 313-340, 2010.

[25] M. B. First et al., Structured Clinical Interview for DSM-IV-TR Axis I Disorders, Research Version, Patient Edition. (SCID-I/P). New York: Biometrics Res., New York State Psychiatric Institute, November 2002.

[26] E. B. Blanchard et al., "Psychometric properties of the ptsd checklist (pcl)," Behaviour res. and therapy, vol. 34, no. 8, pp. 669-673, 1996.

[27] J. Wolpe, The practice of behavior therapy. Pergamon Press New York, 1973.

[28] G. Yannakakis and J. Hallam, "Entertainment modeling through physiology in physical play," International Journal of Human-Computer Studies, vol. 66, no. 10, pp. 741-755, 2008.

[29] M. Kendall, Rank Correlation Methods, 4th ed. London: Griffin, 1970.

[30] G. N. Yannakakis and J. Hallam, "Entertainment modeling through physiology in physical play," International Journal of Human-Computer Studies, vol. 66, no. 10, pp. 741-755, 2008.

[31] M. D. Robinson and G. L. Clore, "Belief and feeling: evidence for an accessibility model of emotional self-report." Psychological bulletin, vol. 128, no. 6, p. 934, 2002.

[32] J. Zhai et al., "Realization of stress detection using psychophysiological signals for improvement of human-computer interactions," in SoutheastCon, 2005. Proceedings. IEEE. IEEE, 2005, pp. 415-420.

[33] M. Benedek and C. Kaernbach, "Decomposition of skin conductance data by means of nonnegative deconvolution," Psychophysiology, vol. 47, no. 4, pp. 647-658, 2010. 